
\title{
28 Research Square \\ One-Year Mortality Prediction in Elderly Patients With Suspected Pneumonia: The Usefulness of a Holistic Approach
}

\section{Alberto Nascè}

Geneva University Hospitals

Astrid Malézieux-Picard

Geneva University Hospitals

Landry Hakiza

Geneva University Hospitals

Thomas Fassier

Geneva University Hospitals

Dina Zekry

Geneva University Hospitals

Nicolas Garin

Riviera Chablais Hospitals

Jérôme Stirnemann

Geneva University Hospitals

Virginie Prendki

Geneva University Hospitals

Xavier Roux ( $\nabla$ xavier.roux@hcuge.ch )

Geneva University Hospitals

\section{Research Article}

Keywords: Pneumonia, elderly, CIRS-G, MNA, CURB-65, FIM, mortality

Posted Date: July 19th, 2021

DOI: https://doi.org/10.21203/rs.3.rs-558655/v1

License: (c) (i) This work is licensed under a Creative Commons Attribution 4.0 International License.

Read Full License 


\section{Abstract \\ Background}

Pneumonia has an impact on long-term mortality in elderly patients. The risk factors associated with poor long-term outcomes are understated. The purpose of this study was to identify the predictors of 1year mortality in older patients having a suspicion of pneumonia, using usual pneumonia severity scores and geriatric evaluation's scores focused on comorbidities, nutritional status and functionality.

\section{Methods}

Consecutive patients over 65 years old and hospitalized with a suspicion of pneumonia were enrolled in a monocentric cohort from May 2015 to April 2016. Three scores were used to assess patients' comorbidities (Cumulative Illness Rating Scale-Geriatric, CIRS-G), malnutrition (Mini Nutritional Assessment, MNA), functionality (Functional Independence Measure, FIM) respectively. Severity of pneumonia was assessed by using the Confusion, Urea, Respiratory Rate, Blood Pressure, and 65-years old score (CURB65), the Pneumonia Severity Index (PSI) and Sequential Organ Failure Assessment score (SOFA). With the exception of CIRS-G, all the scores were obtained prospectively within 48 hours after admission. The main outcome was 1-year mortality. Dates of death were obtained by consulting the cantonal register of deaths. Each score was analysed in univariate and multivariate models and logistic regressions were used to identify contributors to 1-year mortality.

\section{Results}

200 patients were included ( $51 \%$ male, mean age $83.8+/-7.7)$. The 1 -year mortality rate was $30 \%$. Scores associated with 1-year mortality were CURB-65 ( $p<.001)$, SOFA $(p<.05)$, FIM $(p<.01)$, CIRS-G $(p<.001)$ and MNA $(p<.001)$ in univariate analysis. Only CIRS-G $(p<.05)$ and MNA $(p<.05)$ were significant predictors of 1 -year mortality in multivariate analysis.

\section{Conclusions}

Long-term prognosis of pneumonia was poor and we identified that scores assessing comorbidities and malnutrition were important predictors of 1 -year mortality. This should be taken into account for evaluating elderly patients' prognosis, levels and goals of care.

\section{Background}

Pneumonia represents one of the greatest causes of hospitalization and mortality from infection in patients aged 65 or older and a major challenge for physicians ${ }^{1}$. Multiple morbidities and age-related modifications can interfere with the outcome of pneumonia. Effective prognostication at patients' 
admission is therefore required. The two most widely used and validated tools for determining pneumonia 1-month mortality are Pneumonia Severity Index (PSI) ${ }^{2}$ and Confusion, Urea, Respiratory Rate, Blood Pressure, and 65-years old score (CURB-65) ${ }^{3}$. It has also been shown that the Sequential Organ Failure Assessment score and its quick version (SOFA and qSOFA) ${ }^{4-5}$ are good predictors of pneumonia prognosis ${ }^{6}$. However, these scores may have limitations in elderly patients because they don't estimate the biological reserve and systemic functionality, which contribute to short- and long-term prognosis ${ }^{7}$.

Hence, in this population it is important to evaluate comorbidities and to perform a comprehensive geriatric assessment, including the evaluation of their functional, cognitive and nutritional status.

Our research aimed to determine predictors of long-term mortality related to pneumonia in older patients, including commonly used pneumonia and sepsis severity scores and other scores evaluating patients' comorbidities (Cumulative Illness Rating Scale-Geriatric, $\mathrm{CIRS}_{-} \mathrm{G}^{8}$ and Charlson Comorbidity Index, $\mathrm{CCI}^{9}$ ), malnutrition (Mini Nutritional Assessment, $\mathrm{MNA}^{10}$ ), functionality (Functional Independence Measure, $\mathrm{FIM}^{11}$ ) and cognitive status (Mini-Mental State Examination, MMSE ${ }^{12}$ ). We used a prospective observational cohort including patients over 65 years with a suspicion of pneumonia. We hypothesized that the latter predicted better 1-year mortality than pneumonia and sepsis severity scores.

\section{Methods}

\section{STUDY DESIGN AND PARTICIPANTS}

This study took place at a Department of Internal Medicine, Rehabilitation and Geriatrics in Switzerland, a 1,800-bed tertiary care health institution serving a population of about 500,000 inhabitants.

Two hundred consecutive hospitalized patients aged 65 years or older suspected of pneumonia were enrolled in a prospective cohort study between the 1st of May 2015 and the 30th of April 2016, which aimed to determine whether low-dose Computed Tomography (LDCT) had the capacity to enhance the probability of diagnosing pneumonia in elderly patients, and is described elsewhere ${ }^{13}$.

Briefly, the clinical suspicion of community or hospital-acquired pneumonia (defined as an infection developing in 2 or more days after hospital admission) was based, in accordance with the Infectious Disease Society of America/American Thoracic Society (IDSA/ATS) guidelines, on the presence of at least 1 respiratory symptom (new or worsening cough, purulent sputum, pleuritic chest pain, new or worsening dyspnoea, respiratory rate $>20$ breaths/min, auscultatory findings or oxygen saturation $<90 \%$ on room air) and at least 1 clinical or serological finding compatible with pneumonia (body temperature > $38^{\circ} \mathrm{C}$ or $<35^{\circ} \mathrm{C}, \mathrm{CRP}>10 \mathrm{mg} / \mathrm{l}$, white blood cells $>10 \mathrm{G} / \mathrm{l}$ with $>85 \%$ neutrophils or band forms).

Patients who had been treated for a pulmonary infection during the previous 6 months, who had already undergone a CT scan during that specific episode or needed a contrast-enhanced CT, who had to be 
hospitalized in an Intensive Care Unit (ICU), who had received antibiotics for more than 48 hours before inclusion, or patients considered as unable to give their consent were excluded.

\section{DATA COLLECTION}

Data were retrieved from the electronic patient record system. For each included patient, collected data were categorized according to the following domains: demographics, previous treatments, laboratory findings, and comorbidities. The following scores were obtained prospectively within 48 hours after admission: CURB-65, PSI, SOFA, qSOFA, CCI, MNA, MMSE, and FIM. CIRS-G was retrieved retrospectively by two medical doctors and a research nurse. Dates of death were obtained by consulting the institutional database and the cantonal register of deaths. All scores are briefly described in the Appendix.

\section{OUTCOMES}

In this research, we aimed to study the performance of comorbidities (CIRS-G, CCI), malnutrition (MNA), cognitive status (MMSE) and functionality (FIM) assessment tools which were evaluated individually and in a multivariate analysis to predict 1-year mortality in elderly patients hospitalized for suspicion of pneumonia. These were then compared with pneumonia severity scores (CURB-65, PSI), and sepsis severity scores (SOFA and qSOFA).

\section{STATISTICAL ANALYSIS}

Numbers and percentages are reported for categorical variables; medians and interquartile ranges (IQRs) are reported for continuous variables with non-normal distributions, whereas means and standard deviations (SDs) are reported for those continuous variables with a normal distribution.

Categorical variables were compared using the $\chi^{2}$ Test or Fisher Exact Test, while continuous variables were compared using the t-Test or nonparametric Mann-Whitney U Test.

Logistic regression analyses were used to examine associations between long-term mortality and risk factors. In the first step, each risk factor was tested individually. In the second step, excluding prognostic scores, risk factors showing an association in the univariate model $(p<.1)$ were added to multivariate and adjusted models. To compare the accuracy of comorbidity, malnutrition, cognitive status and functionality assessment tools (CIRS-G, CCI, MNA, MMSE and FIM) to predict mortality, the CURB-65 severity score was added into the logistic regression model.

Prognostic values of scores demonstrating an individual capacity to predict 1-year mortality were compared using criteria of sensitivity, specificity, positive and negative likelihood ratios (LR), and diagnostic odds ratios (DOR). The most performing cut-offs were determined using the Youden Index. Receiver Operating Characteristic (ROC) curves were equally performed and Areas Under the Curves (AUC) measured. The Delong test was performed to compare AUC.

Analyses were performed using StataCorp. 2017 (Stata Statistical Software: Release 15. College Station, TX: StataCorp LLC). 
The study was carried out in accordance to the Declaration of Helsinki II Principles (W.M.Association, 2001) and was approved by the local Ethics Committee (CCER 14-250)

\section{Results}

A total of 200 patients were included in our study. Their mean age was $83.8 \pm 7.8$ years old; $51 \%$ were male (Table 1). The overall 1-year mortality rate was $30 \%$. 
Table 1

Baseline characteristics of one-year survivors and non-survivors.

\begin{tabular}{|c|c|c|c|}
\hline Characteristic & $\begin{array}{l}\text { Alive at one year } \\
(N=140)\end{array}$ & $\begin{array}{l}\text { Dead at one year } \\
(N=60)\end{array}$ & $\mathrm{p}$-value \\
\hline Age, mean (SD) & $82.8(7.6)$ & $86.2(7.8)$ & 0.003 \\
\hline Male sex & $68(49,0)$ & $34(56,7)$ & 0.29 \\
\hline Female sex & $72(51,0)$ & $26(43,3)$ & \\
\hline BMI (kg/m2) & $26.1(5.3)$ & $23.3(5.5)$ & 0.0006 \\
\hline \multicolumn{4}{|l|}{ Comorbidities } \\
\hline Diabetes mellitus & $29(21)$ & $11(18)$ & 0.76 \\
\hline COPD & $23(16)$ & $12(20)$ & 0.59 \\
\hline Cancer & $11(8)$ & $6(10)$ & 0.59 \\
\hline CHF & $30(21)$ & $15(25)$ & 0.62 \\
\hline CAD & $20(14)$ & $11(18)$ & 0.47 \\
\hline CVD & $108(77)$ & $48(80)$ & 0.65 \\
\hline Dementia & $26(19)$ & $21(35)$ & 0.012 \\
\hline Chronic Renal Failure & $31(22)$ & $16(27)$ & 0.62 \\
\hline \multicolumn{4}{|l|}{ Severity scores } \\
\hline CURB-65 & $2.1(0.8)$ & $2.6(0.9)$ & 0.0002 \\
\hline PSI & $106.7(23.6)$ & $113.0(33.5)$ & 0.07 \\
\hline SOFA & $2.3(1.4)$ & $2.9(2.1)$ & 0.014 \\
\hline qSOFA & $0.85(0.6)$ & $1.02(0.8)$ & 0.064 \\
\hline \multicolumn{4}{|l|}{ Geriatric scores } \\
\hline FIM $(N=171)$ & $76.3(32.0)$ & $64.5(26.0)$ & 0.009 \\
\hline Charlson & $2.96(1.9)$ & $3.28(2.2)$ & 0.17 \\
\hline
\end{tabular}

Data presented as $n(\%)$ or mean (SD). The number of patient $(\mathrm{N})$ is 200 unless otherwise stated. BMI: Body Mass Index; CAD: Coronary Artery Disease; CIRS-G : Cumulative Illness Rating Scale-Geriatric; CHF: Chronic Heart Failure; COPD: Chronic Obstructive Pulmonary Disease; CRP: C-Reactive Protein; CURB-65: Confusion Urea Respiratory rate Blood pressure 65-years old; CVD: Cardio-Vascular Disease; FIM: Functional Independence Measure; FiO2: Fraction of Inspired Oxygen; MMSE: Mini Mental State Examination; MNA: Mini Nutritional Assessment; NT-proBNP: N-Terminal prohormone of Brain Natriuretic Peptide; PaO2: Partial pressure of Arterial blood Oxygen; PSI: Pneumonia Severity Index; SOFA: Sequential Organ Failure Assessment; qSOFA: Quick Sequential Organ Failure Assessment. 


\begin{tabular}{|c|c|c|c|}
\hline Characteristic & $\begin{array}{l}\text { Alive at one year } \\
(N=140)\end{array}$ & $\begin{array}{l}\text { Dead at one year } \\
(N=60)\end{array}$ & p-value \\
\hline CIRS-G & $22.2(6.5)$ & $25.5(5.2)$ & 0.0003 \\
\hline MNA $(N=178)$ & $8.9(2.6)$ & $6.8(3.0)$ & $<0.0001$ \\
\hline MMSE $(\mathrm{N}=162)$ & $23(6.1)$ & $19(6.7)$ & 0.0007 \\
\hline \multicolumn{4}{|l|}{ Laboratory findings } \\
\hline Urea (mmol/l) & $8.9(4.8)$ & $10.4(6.4)$ & 0.033 \\
\hline Albumin $(\mathrm{g} / \mathrm{l})(\mathrm{N}=193)$ & $35.2(5.3)$ & $34.2(5.7)$ & 0.13 \\
\hline $\mathrm{PaO} 2(\mathrm{kPa})$ & $10.2(4.3)$ & $10.7(5.2)$ & 0.25 \\
\hline FiO2 & $0.27(0.12)$ & $0.30(0.14)$ & 0.10 \\
\hline Hemoglobin $(\mathrm{g} / \mathrm{l})$ & $123(19.3)$ & $124(16.9)$ & 0.30 \\
\hline Leukocytes (G/I) & $11.7(4.8)$ & $11.1(5.9)$ & 0.24 \\
\hline CRP (mg/l) & $128(103)$ & $94(73)$ & 0.01 \\
\hline NT-proBNP $(\mathrm{ng} / \mathrm{l})(\mathrm{N}=170)$ & $2537(2651)$ & 3402 (3032) & 0.035 \\
\hline \multicolumn{4}{|c|}{$\begin{array}{l}\text { Data presented as } \mathrm{n}(\%) \text { or mean (SD). The number of patient (N) is } 200 \text { unless otherwise stated. BMI } \\
\text { Body Mass Index; CAD: Coronary Artery Disease; CIRS-G : Cumulative Illness Rating Scale-Geriatric; } \\
\text { CHF: Chronic Heart Failure; COPD: Chronic Obstructive Pulmonary Disease; CRP: C-Reactive Protein; } \\
\text { CURB-65: Confusion Urea Respiratory rate Blood pressure 65-years old; CVD: Cardio-Vascular Disease } \\
\text { FIM: Functional Independence Measure; FiO2: Fraction of Inspired Oxygen; MMSE: Mini Mental State } \\
\text { Examination; MNA: Mini Nutritional Assessment; NT-proBNP: N-Terminal prohormone of Brain } \\
\text { Natriuretic Peptide; PaO2: Partial pressure of Arterial blood Oxygen; PSI: Pneumonia Severity Index; } \\
\text { SOFA: Sequential Organ Failure Assessment; qSOFA: Quick Sequential Organ Failure Assessment. }\end{array}$} \\
\hline
\end{tabular}

Univariate analysis found that the significant factors contributing to 1-year mortality were age, Body Mass Index (BMI), urea, NT-proBNP and CURB-65, SOFA, FIM, CIRS-G, MNA and MMSE scores. Patients who died at one year were significantly older and had a lower $B M I(p<.005$ and $p<.001$ respectively). No significant differences were found in terms of sex and other comorbidities. Mean CURB-65 score was significantly higher $(p<.001)$ in patients who died at one year $(2.6 \pm 0.9)$ than in patients alive $(2.1 \pm 0.8)$. However, no significant difference was found for PSI or qSOFA scores. Mean SOFA score was higher in deceased patients $(2.9 \pm 2.1)$ than in survivors $(2.3 \pm 1.4)(p<.05)$. Mean FIM score was significantly lower in patients who died $(76.3 \pm 32$ vs $64.5+/-26 ; p<.01)$. The deceased group had a mean CIRS-G score of $25.5 \pm 5.2$ compared with a mean score of $22.2 \pm 6.5$ amongst surviving patients $(p<.001)$. Lower MNA ( $6.8 \pm 3.0$ vs $8.9 \pm 2.6)$ and MMSE $(19 \pm 6.7$ vs $23 \pm 6.1)$ scores were found to be independently associated with a worse 1 -year prognosis $(p<.001)$. With regards to laboratory exams, we found a statistical correlation between levels of urea $(10.4 \pm 6.4 \mathrm{mmol} / \mathrm{l} v \mathrm{vs} 8.9 \pm 4.8 \mathrm{mmol} / \mathrm{l} ; \mathrm{p}<.05)$ and NT-proBNP $(3402 \pm 3032 \mathrm{ng} / \mathrm{l}$ vs $2537 \pm 2651 \mathrm{ng} / \mathrm{l} ; \mathrm{p}<.05)$ and mortality rates. In multivariate analysis, 
only CIRS-G and MNA were found to be significant contributors to the 1-year mortality $(p<.05)$ (Tables 2$3, A 1$ and $A 2)$.

Table 2

Association between scores evaluating comorbidities, malnutrition and functionality and 1-year mortality, after CURB-65 adjustment (multivariate analysis).

\begin{tabular}{|llll|}
\hline Variable & OR & $95 \% \mathbf{C l}$ & $\mathbf{p}$ \\
\hline CIRS-G & 1.08 & $1.01-1.15$ & 0.014 \\
\hline MNA & 0.83 & $0.71-0.96$ & 0.012 \\
\hline MMSE & 0.94 & $0.87-1.0$ & 0.068 \\
\hline FIM & 0.99 & $0.97-1.0$ & 0.269 \\
\hline Charlson & 1.15 & $0.95-1.39$ & 0.136 \\
\hline
\end{tabular}

Table 3

Association between pneumonia/sepsis severity scores and scores evaluating comorbidities, malnutrition and functionality.

\begin{tabular}{|lll|}
\hline Score & 1-month mortality & 1-year mortality \\
\hline CURB-65 & $1.78(p=0.1)$ & $1.85(p=0.004)$ \\
\hline SOFA & $1.3(p=0.81)$ & $1.24(p=0.048)$ \\
\hline CIRS-G & $1.07(p=0.24)$ & $1.08(p=0.021)$ \\
\hline MNA & $0.92(p=0.53)$ & $0.81(p=0.007)$ \\
\hline $\begin{array}{l}\text { Results of association between CURB-65, SOFA, CIRS-G and MNA scores and 1-month and 1-year } \\
\text { mortality (multivariate analysis). Results expressed as Odds Ratios (OR). }\end{array}$ \\
\hline
\end{tabular}

The performance of all the scores proving to independently predict 1-year mortality were tested. Sensitivity and specificity were $59 \%$ and $70 \%$ for CIRS-G $\geq 26,71 \%$ and $65 \%$ for MNA $\geq 8,55 \%$ and $70 \%$ for CURB- $65 \geq 3$, and $52 \%$ and $58 \%$ for SOFA $\geq 3$, respectively (Table 4). The Areas Under the Curves (AUC) values were 0.70 (95\% Cl 0.63-0.77) for MNA, 0.66 (95\% Cl 0.58-0.72) for CIRS-G, $0.64(95 \% \mathrm{Cl}$ 0.57-0.71) for CURB-65, and 0.57 (95\% $\mathrm{Cl} 0.48-0.66)$ for SOFA scores (Fig. 1 and Table 4). No significant differences were found when we compared the AUC (results not shown). 
Table 4

Comparison of diagnostic value of CURB-65, CIRS-G, MNA and SOFA scores.

\begin{tabular}{|llllll|}
\hline Variable & Sensitivity & Specificity & LR+ & LR- & DOR(LR+/LR-) \\
\hline CURB-65 $\geq 3$ & 0.55 & 0.70 & 1.83 & 0.64 & 2.85 \\
\hline CIRS-G $\geq 26$ & 0.59 & 0.70 & 1.94 & 0.59 & 3.27 \\
\hline MNA $\geq 8$ & 0.71 & 0.65 & 2.0 & 0.45 & 4.46 \\
\hline SOFA $\geq 3$ & 0.52 & 0.58 & 1.24 & 0.83 & 1.49 \\
\hline $\begin{array}{l}\text { Sensitivity, specificity, positive and negative likelihood ratio and diagnostic odds ratio (DOR) values } \\
\text { for one-year mortality according to CIRS-G, MNA, SOFA and CURB-65 at the best cut-off values } \\
\text { (computed with Youden index) }\end{array}$ &
\end{tabular}

\section{Discussion}

To our knowledge, the present study is the first to compare the prognostic value of both pneumonia and sepsis severity scores and scores evaluating comorbidities, malnutrition, cognitive status and functionality in predicting 1-year mortality in an elderly population hospitalized with a suspicion of pneumonia.

In our study, CURB-65 and SOFA were the only pneumonia and sepsis severity scores to prognosticate 1year mortality. Among tools performed for the comprehensive geriatric assessment at patients' admission, CIRS-G, MNA, and FIM evaluating comorbidity, malnutrition and functionality, were strong predictors of 1-year mortality.

Many studies reported a series of risk factors associated with long-term mortality in patients suffering from pneumonia ${ }^{7}$. Indeed, pulmonary infections may have significant impacts on various organ systems, such as respiratory, cardiovascular, and neurological ones, leading to the potential worsening of preexisting comorbidities and subsequent higher fatality rates ${ }^{7}$. Therefore, a better understanding of longterm mortality prediction, measured at $30 \%$ in our study, seems urgent.

Amongst the risk factors commonly associated with poor long-term outcomes, we investigated the role of comorbidities, malnutrition, functionality and dementia. Concerning the latter, its contribution to elderly patients' prognosis has been studied by Uranga et al. In their research, dementia was found to be the best predictor of one-year mortality in patients hospitalized with community-acquired pneumonia ${ }^{14}$. Similar results were found in a meta-analysis by Foley et al who showed that the odds of pneumonia-associated mortality increased more than 2 -fold in patients with cognitive disorders ${ }^{15}$. We found that cognitive disorders were associated with poorer 1-year outcomes but only in univariate analysis.

Another aspect often playing a role in elderly patients' mortality is malnutrition. The MNA was developed as a nutritional screening tool. Using this tool, we were able to identify a very strong correlation between malnutrition and poor outcomes at one year, indicating that assessment of the nutritional status at 
admission may help in reducing elderly patients' mortality. Few other studies detected similar results. Yoon et $\mathrm{al}^{16}$, studying an elderly population with aspiration pneumonia, identified lower BMI and hypoalbuminemia as independent prognostic factors for 5 -year mortality. Yeo et al ${ }^{17}$ recently highlighted that malnutrition was strongly linked with higher 2-year mortality in people suffering from pneumonia, particularly in the elderly, making essential a routine nutritional assessment at admission. Among elderly patients who have recovered from pneumonia, those who are malnourished have an increased risk of developing impaired muscle and respiratory function, which may lead to more severe long-term outcomes $^{18}$.

Regarding comorbidities, we took into consideration the $\mathrm{CCl}$ and the CIRS-G. One of our main findings was a strong correlation between the CIRS-G and mortality at one year. Similar mortality results, although not focused on a specific disease, were found in the recent literature. A systematic review on the performance of different morbidity scores to predict mortality in inpatients hospitalized for any medical condition showed that CIRS-G, as per 1 point increase, was significantly associated with post-discharge mortality ${ }^{19}$. Zekry et al highlighted in patients hospitalized in an acute geriatric hospital that CIRS-G provided the most accurate risk prediction for 5-year mortality among six widely used multimorbidity scores ${ }^{20}$. Salvi et al confirmed the validity of the CIRS-G as an indicator of health status and demonstrated its ability to predict 18-month mortality and rehospitalisation amongst elderly inpatients ${ }^{21}$. In the same line, Ritt et al showed that CIRS-G proved accurate in forecasting 1-year mortality in elderly patients $^{22}$. In alignment with the limitation of the Charlson Comorbidity Index, which is considered to be potentially misleading in rating elderly patients' multimorbidity and not sufficiently able to predict longterm prognosis in geriatric populations ${ }^{19-20}$, we did not find any relevant correlation between this score and patients' 1-year mortality.

Amongst several scales estimating patients' dependence and functionality ${ }^{23-25}$, we decided to use the FIM, which in a recent study among critically ill elderly patients admitted to an intermediate care unit proved a correlation between low ratings on the scale and higher 1-year mortality rates ${ }^{26}$. Another research showed that frailty, defined as unintentional weight loss, self-reported exhaustion, weakness slow walking speed, and low physical activity, was strongly associated with the severity of pneumonia and a higher 1-year mortality in older patients, suggesting that frailty should be detected early to improve their management ${ }^{27}$. In our findings, lower FIM ratings were individually associated with a poorer longterm prognosis but failed in finding significant 1-year mortality correlations in multivariate models.

We investigated the ability of the two most widely used prognostic tools (CURB-65 and PSI), validated as 30-day pneumonia mortality predictors, to determine long-term prognosis amongst a population of elderly people. We did not find any association between the PSI and the prognosis of our patients. Interestingly, CURB-65 was an efficient long-term mortality predictor in our cohort. Similar results were found by Wesemann et $\mathrm{al}^{28}$ in a predominantly older population. 
It has recently been shown that SOFA and qSOFA are useful scores for evaluating pneumonia mortality in geriatric populations ${ }^{29-31}$. In our study, we found a weak but significant correlation between SOFA scores at admission and 1-year mortality rates, probably due to a persistent impact of organ failure caused by pneumonia on frail people's homeostasis. No significant association was found between the qSOFA and long-term prognosis.

The present study's main strength was the consecutive inclusion of elderly patients hospitalized and treated for a suspicion of pneumonia. It has several limitations, however. As a single-centre study carried out with a relatively small number of patients, it should not be generalized to other hospitals. Since we focused our attention on elderly individuals with suspected pneumonia, our results should not be exported to other clinical contexts. Additionally, CIRS-G was retrieved retrospectively from medical records, exposing our analysis to potentials biases. Finally, we did not record the 'Do not resuscitate' orders in our cohort, which might affect patients' outcomes and be a source of bias.

\section{Conclusions}

The CIRS-G and MNA were found to be promising prognostic tools for assessing long-term mortality in a cohort of elderly people suffering from suspected pneumonia. Apart from CURB-65 and SOFA, no other pneumonia severity nor sepsis scores could efficiently predict 1-year mortality. Our findings suggest that a more holistic approach, including nutritional and comorbidity assessments, should be performed when treating older patients with a suspicion of pneumonia and discussing about their prognosis and goals of care. Nevertheless, further studies are needed to evaluate the concrete impact of managing comorbidities and malnutrition on long-term prognosis.

\section{List Of Abbreviations}

CIRS-G

Cumulative Illness Rating Scale-Geriatric

MNA

Mini Nutritional Assessment

MIF

Functional Independence Measure

CURB65

Confusion, Urea, Respiratory Rate, Blood Pressure, and 65-years old score

PSI

Pneumonia Severity Index

SOFA

Sequential Organ Failure Assessment score

$\mathrm{CCl}$

Charlson Comorbidity Index

MMSE 
Mini-Mental State Examination

CT

Computed Tomography

LDCT

low-dose Computed Tomography

IDSA

/ATS Infectious Disease Society of America/

ATS

American Thoracic Society

ICU

Intensive Care Unit

IQRs

interquartile ranges

SDs

standard deviations

LR

likelihood ratios

DOR

diagnostic odds ratios

ROC

Receiver Operating Characteristic

AUC

Areas Under the Curves

BMI

Body Mass Index

\section{Declarations}

\section{Ethics approval and consent to participate:}

This study was conducted in accordance with the principles of the Declaration of Helsinki and was approved by the Geneva Ethics Committee ( $\left.{ }^{\circ} 2018.00218\right)$. All methods were carried out in accordance with relevant guidelines and regulations. No administrative permissions were required to access and use the medical records, according to the Swiss law regarding the human medical research (law relating to research on humans with the exception of clinical trials, ordinance on research involving humans / 2013 September the $20^{\text {th }}$ and law of September 30, 2011 on research on human beings). The need for consent was waived by the Institutional Review Boards (IRBs) of Geneva and the Geneva Ethics Committee.

\section{Consent for publication}

Not applicable 
Availability of data and materials

The datasets generated during and/or analysed during the current study are available from the corresponding author on reasonable request.

\section{Competing interests}

The authors declare that they have no competing interests

\section{Funding}

The study was funded by grants from the Geneva University Hospitals (HUG) (Research \& Development Grant, Medical Directorate, HUG and Research Fund of the Department of Internal Medicine of the University Hospital and the Faculty of Medicine of Geneva, this Fund receives an unrestricted grant from AstraZeneca Switzerland) and the Ligue Pulmonaire Genevoise, a non-profit association involved in the care of patients with respiratory diseases. Funding was use to remunarate a reeach nurse aiming to collect data and to fill the case reports forms. The funders had no role in the collection data process, data analysis and did not participate for the manuscript wrtiting.

\section{Authors' contributions}

AN designed the study, analysed data and drafted the manuscript. AM read the manuscript, analysed data and amended the manuscript. LH contributed to collect data, read and amended the manuscript. TF made the interpretation of data, substantively revised the manuscript, read and corrected the final manuscript. DZ made substantial corrections to the manuscript and corrected the final manuscript. NG substantively revised the manuscript, analysed data and performed stastistical tests, read and corrected the final manuscript. JS: was a major contributor in statistical analysis, collected data, read and corrected the final manuscript. VP collected data, included patients, designed the study, amended the manuscript with major contribution, performed statistical analysis. XR collected data, included patients, designed the study, amended the manuscript with major contribution, performed statistical analysis. Alberto Nascè and Astrid Malézieux-Picard contributed equally to this work. Virginie Prendki and Xavier Roux contributed equally to this work.

All authors read and approved the final manuscript.

\section{Acknowledgements}

None.

\section{References}

1. Welte T, Torres A, Nathwani D. Clinical and economic burden of community-acquired pneumonia among adults in Europe. Thorax 2012;67(1):71-79. 
2. Fine MJ, Auble TE, Yealy DM et al. A prediction rule to identify low-risk patients with communityacquired pneumonia. N Engl J Med 1997; 336(4):243-250.

3. Lim WS, van der Eerden MM, Laing $\mathrm{R}$ et al. Defining community acquired pneumonia severity on presentation to hospital: an international derivation and validation study. Thorax 2003;58(5):377382.

4. Vincent JL, Moreno R, Takala J et al. The SOFA (Sepsis-related Organ Failure Assessment) score to describe organ dysfunction/failure. On behalf of the Working Group on Sepsis-Related Problems of the European Society of Intensive Care Medicine. Intensive Care Med 1996;22(7):707-10

5. Seymour CW, Liu VX, Iwashyna TJ et al. Assessment of Clinical Criteria for Sepsis: For the Third International Consensus Definitions for Sepsis and Septic Shock (Sepsis-3). JAMA 2016;315(8):762-774.

6. Asai N, Watanabe H, Shiota A et al. Efficacy and accuracy of qSOFA and SOFA scores as prognostic tools for community-acquired and healthcare-associated pneumonia. Int J Infect Dis 2019;84:89-96

7. Restrepo MI, Faverio P, Anzueto A. Long-term prognosis in community-acquired pneumonia. Curr Opin Infect Dis 2013;26(2):151-158.

8. Parmelee PA, Thuras PD, Katz IR et al. Validation of the cumulative illness rating scale in a geriatric residential population. J Am Geriatr Soc 1995;43(2):130-137

9. Charlson ME, Pompei P, Ales KL et al. A new method of classifying prognostic comorbidity in longitudinal studies: Development and validation. J Chron Dis 1987;40(5):373-383.

10. Guigoz Y, Vellas B, Garry PJ. Assessing the nutritional status of the elderly: the Mini Nutritional Assessment as part of the geriatric evaluation. Nutr Rev 1996;54:S59-65.

11. Kidd D, Stewart G, Baldry J et al. The functional independence measure: a comparative validity and reliability study. Disabil Rehabil. 1995;17(1):10-4.

12. Folstein M, Folstein SE, McHugh PR. 'Mini-mental state': a practical method for grading the cognitive state of patients for the clinician. Journal of Psychiatric Research 1975;12(3):189-198

13. Prendki V, Scheffler M, Huttner B et al. Low-dose computed tomography for the diagnosis of pneumonia in elderly patients: a prospective, interventional cohort study. Eur respir J 2018;51(5):170237.

14. Uranga A, Quintana JM, Aguirre U et al. Predicting 1-year mortality after hospitalization for community-acquired pneumonia. PloS One 2018;13(2)e:0192750

15. Foley NC, Affoo RH, Martin RE. A systematic review and meta-analysis examining pneumoniaassociated mortality in dementia. Dement Geriatr Cogn Disord 2015;39(1-2):52-67.

16. Yoon HY, Shim SS, Kim SJ et al. Long-Term Mortality and Prognostic Factors in Aspiration Pneumonia. J Am Med Dir Assoc. 2019 Sep;20(9):1098-1104.

17. Yeo HJ, Byun KS, Han J et al. Prognostic significance of malnutrition for long-term mortality in community-acquired pneumonia: a propensity score matched analysis. Korean J Intern Med 2019;34(4):841-849. 
18. Chebib N, Cuvelier C, Malézieux-Picard A et al. Pneumonia prevention in the elderly patients: the other sides. Aging Clin Exp Res 2019 Dec 31. doi: 10.1007/s40520-019-01437-7.

19. Soh $\mathrm{CH}, \mathrm{UI}$ Hassan SW, Sacre J et al. Morbidity measures predicting mortality in inpatients: a systematic review. J Am Med Dir Assoc 2020;21(4):462-468.

20. Zekry D, Valle BH, Michel JP et al. Prospective comparison of six comorbidity indices as predictors of 5 years post hospital discharge survival in the elderly. Rejuvenation Res 2010;13(6):675-682.

21. Salvi F, Miller MD, Grilli A et al. A manual of guidelines to score the modified cumulative illness rating scale and its validation in acute hospitalized elderly patients. J Am Geriatr Soc 2008;56(10):192631.

22. Ritt M, Ritt Jl, Sieber CC et al. Comparing the predictive accuracy of frailty, comorbidity, and disability for mortality: a 1-year follow-up in patients hospitalized in geriatric wards. Clin Interv Aging 2017;12:293-304

23. Mahoney Fl, Barthel DW. Functional evaluation: the Barthel Index. Md State Med J 1965;14:61-65.

24. Mitnitski AB, Mogilner AJ, Rockwood K. Accumulation of deficits as a proxy measure of aging. ScientificWorldJournal 2001;1:323-36.

25. Rockwood K, Song X, MacKnight $C$ et al. A global clinical measure of fitness and frailty in elderly people. CMAJ 2005;173(5):489-95

26. D'Andrea A, Le Peillet $D$, Fassier T et al. Functional Independence Measure score is associated with mortality in critically ill elderly patients admitted to an intermediate care unit. BMC Geriatr 2020;20(1):334

27. Luo J, Tang W, Sun Y et al. Impact of frailty on 30-day and 1-year mortality in hospitalised elderly patients with community-acquired pneumonia: a prospective observational study. BMJ Open 2020;10(10):e038370.

28. Wesemann T, Nüllmann H, Pflug MA et al. Pneumonia severity, comorbidity and 1-year mortality in predominantly older adults with community-acquired pneumonia: a cohort study. BMC Infect Dis 2015;15:2.

29. Cillóniz C, Dominedò C, lelpo A et al. Risk and prognostic factors in very old patients with sepsis secondary to Community-Acquired Pneumonia. J.Clin.Med 2019;8(7):961

30. Zhou H, Lan T, Guo S. Stratified and prognostic value of admission lactate and severity scores in patients with community-acquired pneumonia in emergency department: A single-center retrospective cohort study. Medicine (Baltimore). 2019;98(41):e17479.

31. Zhang X, Liu B, Liu $Y$ et al. Efficacy of the quick sequential organ failure assessment for predicting clinical outcomes among community-acquired pneumonia patients presenting in the emergency department. BMC Infect Dis 2020;20(1):316.

\section{Figures}




\section{a: CURB-65}

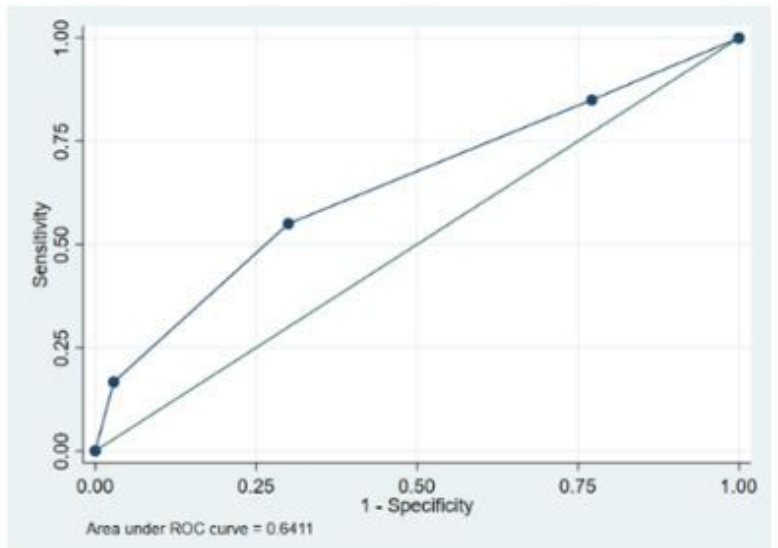

\section{b: SOFA}

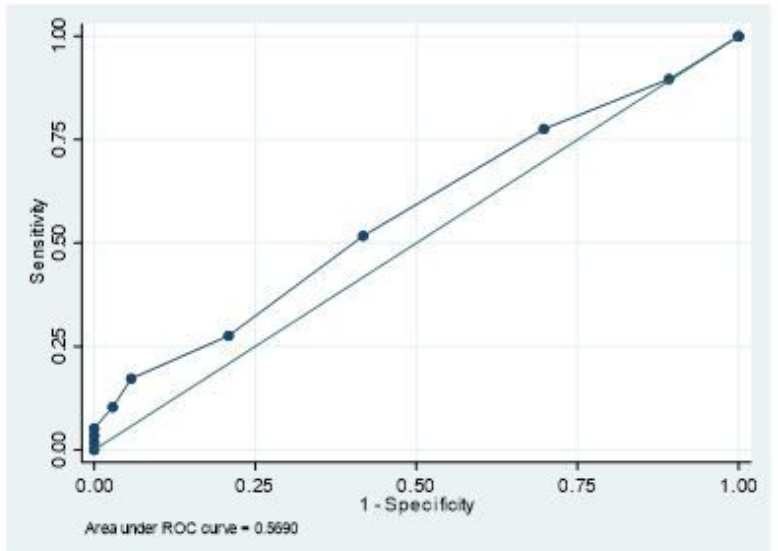

\section{c: MNA}

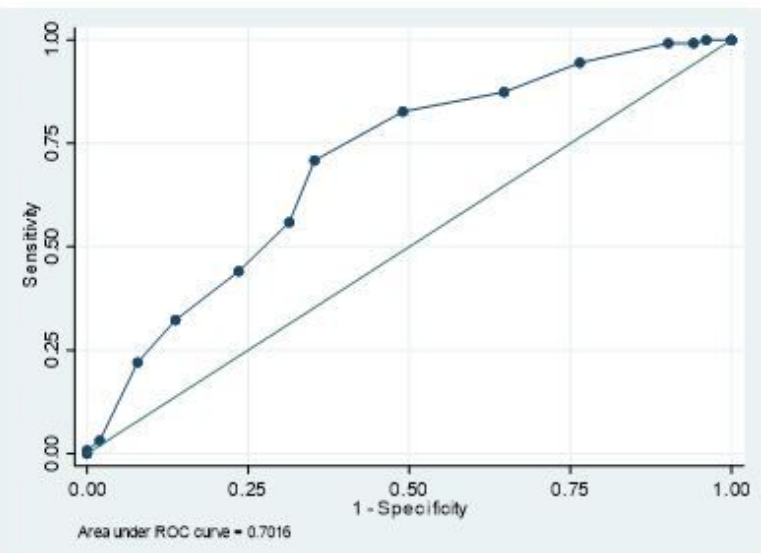

d: CIRS-G

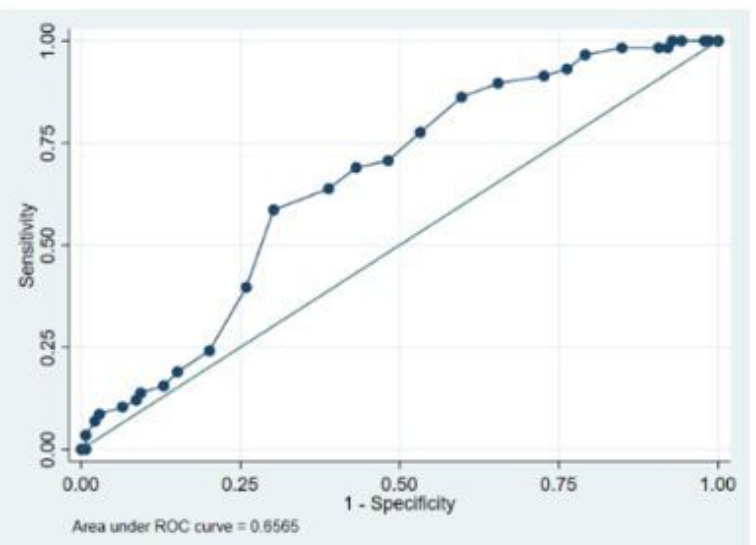

\section{Figure 1}

ROC (Receiver Operating Characteristic) curves for pneumonia one-year mortality: a) CURB65 Area Under the Curve $(95 \% \mathrm{Cl})=0.64$. Optimal cut-off point: $3 ;$ b) SOFA Area Under the Curve $(95 \% \mathrm{Cl})=0.57$. Optimal cut-off point: 3, c) MNA Area Under the Curve $=0.70$. Optimal cut-off point: 8; d) CIRS-G Are under the Curve $=0.66$. Optimal cut-off point: 26 .

\section{Supplementary Files}

This is a list of supplementary files associated with this preprint. Click to download.

- BMCgAppendixv2.docx 